\title{
Acid clearance and oesophageal sensitivity in patients with progressive systemic sclerosis
}

\author{
G Basilisco, R Barbera, M Molgora, M Vanoli, P Bianchi
}

\begin{abstract}
This study examined the hypothesis that impaired oesophageal peristalsis was associated with delayed oesophageal clearance of acid in patients with progressive systemic sclerosis (PSS), some of whom are thought to have impaired oesophageal sensitivity to acid. Sixteen patients with PSS had: (a) oesophageal manometry and endoscopy; (b) acid perfusion of the oesophagus with simultaneous measurement of intraoesophageal $\mathrm{pH}$ during perfusion and for the next 10 minutes; (c) 22 hour monitoring of intraoesophageal pH; and (d) an evaluation of reflux symptoms during and after perfusion and during overnight $\mathrm{pH}$ monitoring. By oesophageal manometry, eight patients had normal peristalsis and eight patients had impaired peristalsis. Oesophageal endoscopy was unremarkable in patients with normal peristalsis, whereas all patients with impaired peristalsis had oesophagitis. The time needed to clear the oesophagus of perfused acid was shorter $(p<0.01)$ in patients with normal peristalsis and acid clearance time was significantly correlated $(p<0.01)$ with acid exposure time during overnight pH monitoring. During and after oesophageal perfusion, the nature, duration, and severity of symptoms did not differ between the groups, but overnight symptoms lasted longer $(p<0.05)$ in patients with impaired peristalsis. It is concluded that in PSS: (1) Impaired oesophageal motility delayed the clearance of acid and increased the exposure time to acid. (2) Acid clearance time is a useful parameter of impaired oesophageal motor function. The assessment of acid clearance time can be used as an alternative to overnight pH monitoring, to assess the impairment of oesophageal acid clearance. (3) Oesophageal sensitivity to acid was preserved in patients with impaired peristalsis and oesophagitis. (4) Reflux symptoms lasted longer in patients with prolonged oesophageal acid exposure but were still reported for a small fraction of the total acid exposure time. Thus, reflux symptoms reflect poorly prolonged exposure of the oesophagus to acid and are not a reliable guide to acid injury of the oesophagus in PSS.

(Gut 1993; 34: 1487-1491)
\end{abstract}

In progressive systemic sclerosis (PSS), oesophageal peristalsis may be impaired, ${ }^{1}$ oesophageal acid exposure time prolonged, ${ }^{2}$ oesophageal transit of a radiolabelled liquid meal delayed, ${ }^{3}$ and oesophagitis is common. ${ }^{2}$ In addition, the duration of acid reflux is longer in PSS patients with oesophagitis than in patients with a similar degree of oesophagitis but with no connective tissue disease. ${ }^{4}$ Moreover, lower oesophageal sphincter pressure is often severely reduced in PSS $^{24}$ and gastric emptying delayed, ${ }^{5}$ both these phenomena could aggravate gastro-oesophageal reflux. ${ }^{6}$ The prolonged duration of reflux seen during $\mathrm{pH}$ monitoring ${ }^{2}$ or after a meal ${ }^{4}$ in PSS could thus be related to defective clearance or to a greater quantity of reflux. No data are available, however, in PSS on the time taken by the weakened oesophagus to clear itself of a known amount of acid. We proposed that acid clearance time would be delayed in patients with impaired peristalsis and that the delay in clearance would be correlated with acid exposure time.

Although heartburn is the most common oesophageal symptom in patients with $\mathrm{PSS},{ }^{27}$ a reduced sensitivity to acid, related to the destruction of oesophageal sensory pathways, has been suggested. ${ }^{7}$ This hypothesis was based on the finding of only a $30 \%$ positive response to acid perfusion in a series of 53 PSS patients. ${ }^{7}$ Peripheral neuropathy has also been seen in some patients with PSS $^{8-12}$ and neuropathy has been implicated in the initial changes in oesophageal motility. ${ }^{13}$ We speculated, therefore, that oesophageal sensitivity to acid would be reduced in PSS patients with impaired oesophageal peristalsis.

Our aims in this study were to assess in PSS patients, with or without impaired oesophageal peristalsis, acid clearance by the oesophagus and oesophageal sensitivity to acid.

\section{Patients and methods}

We studied 16 unselected patients with PSS (mean age 47 years, range $26-62,15$ women, one man) referred to our gastroenterology unit in the period from September 1991 to April 1992 for the assessing of oesophageal involvement. The diagnosis of PSS was based on the preliminary criteria of the American Rheumatism Association. ${ }^{14}$ One major or two minor criteria were required for the diagnosis; the major criterion was scleroderma affecting the digits, forearms or face (15 patients); minor criteria were sclerodactyly alone (10 patients), distal pitting scars of finger tips (10 patients), loss of substance of the distal finger pads (nine patients) and bilateral pulmonary fibrosis (seven patients). Raynaud's phenomenon was reported by all patients, for a mean (range) time of 10.5 years (1 to 23).

Patients completed a standard questionnaire for the presence or absence during the preceding month of dysphagia, heartburn, regurgitation, nausea, or vomiting. All had endoscopy to determine the presence or absence of macroscopic oesophagitis (grade 1: erythema or linear erosions; grade 2: confluent erosions or ulcera- 
tions; grade 3: circumferential erosions or ulcerations; strictures).

Oesophageal manometry and acid perfusion tests were performed in the morning, with the fasting patient in the left lateral position. For 24 hours all abstained from drugs that could interfere with oesophagogastric function. Oesophageal manometry was performed as described by Richter et al. ${ }^{15}$ An 8 lumen polyvinyl catheter (outer diameter $4.5 \mathrm{~mm}$; inside diameter of each lumen $0.8 \mathrm{~mm}$; Arndorfer Specialties Inc, Greendale, USA) was passed through the nose or mouth and continuously perfused using a low compliance pneumohydraulic capillary perfusion system (Arndorfer Specialties Inc, Greendale, USA) at a rate of $0.5 \mathrm{ml} /$ minute. Pressures were transmitted to external pressure transducers and recorded on a polygraph. With the distal four openings spaced $1 \mathrm{~cm}$ apart at 90 degrees, lower oesophageal sphincter pressure was recorded once by station pull through $(0.5$ $\mathrm{cm}$ increments/30 seconds) and twice by rapid $(1 \mathrm{~cm} / 1$ second) pull through. The mean station pull through pressure was calculated as the average of four separate pressure values measured from the gastric baseline to the midpoint of respiratory variations. Mean rapid pull through pressure was calculated as the average of eight separate pressure values measured from the gastric baseline to the peak of the pressure rise,

The catheter was then positioned to record motility $3,8,13$, and $18 \mathrm{~cm}$ above the upper border of the lower oesophageal sphincter. Ten wet ( $5 \mathrm{ml}$ of water) swallows were performed at intervals of at least 60 seconds. Amplitude and duration of contractions at each recording site were calculated as the average of 10 swallows. Amplitude was measured from the intraoesophageal baseline to the peak of the pressure wave; duration was measured from the point where the upstroke of the contraction left the baseline to the point where the downstroke of the contractions returned to baseline. The individual values for the recording sites 3 and $8 \mathrm{~cm}$ above the lower oesophageal sphincter were combined and designated as distal oesophageal contractile amplitude and duration. Peristalsis was defined as impaired when the distal oesophageal contractile amplitude was $<20 \mathrm{~mm} \mathrm{Hg}$.

After oesophageal manometry, with the manometric catheter still in place, an antimony electrode mounted on a polyvinyl catheter (outer diameter $2 \cdot 1 \mathrm{~mm}$; Monocristant 0011, Synectics Medical, Stockholm) was passed through the nose and positioned in the oesophagus $5 \mathrm{~cm}$ above the upper border of the lower oesophageal sphincter. A reference electrode was applied on the skin of the abdomen. Both electrodes were connected to a pH recorder that stored data every four seconds. A constant perfusion $(8 \mathrm{ml} /$ minute) of saline (five minutes) followed by $\mathrm{HCl}$ $0 \cdot 1 \mathrm{~N}$ (10 minutes) was given by an infusion pump (Watson and Marlow Ltd) through the manometry catheter, with an open tip placed $5 \mathrm{~cm}$ above the lower oesophageal sphincter. The time needed to empty the dead space of the infusion was set (mean (SEM) of six replications) at 95 (4) seconds. Patients were allowed to swallow during and after perfusion. Intraoeso- phageal $\mathrm{pH}$, and the intensity and duration of symptoms (heartburn, acid regurgitation) were recorded during infusions and for the next 10 minutes. Acid clearance time was defined as the interval between the end of acid infusion and the time at which the intraluminal $\mathrm{pH}$ first rose above four.

After 10 minutes of infusion, the manometric catheter was removed, two sips of tap water were given to clear acid from the oesophagus, and 22 hours of $\mathrm{pH}$ monitoring were started. Patients reported the intensity and duration of symptoms with a diary card and the onset of symptoms with an event market on the $\mathrm{pH}$ recording. Patients were also instructed to eat at $1 \mathrm{pm}$ and $8 \mathrm{pm}$ and to remain upright during the day and to be supine from $10 \mathrm{pm}$ to $8 \mathrm{am}$.

At the end of the recording, $\mathrm{pH}$ data were transferred to a computer for analysis. Acid gastro-oesophageal reflux was defined as a fall in $\mathrm{pH}<4$ for at least five seconds from a $\mathrm{pH}>5$. The percentage of time at $\mathrm{pH}<4$, the minutes at $\mathrm{pH}<4$, the number of episodes of reflux, those lasting longer than five minutes, and the duration of the longest period of reflux were calculated with Esophogram 9802 Version 5.10 (Synectics Medical, Stockholm). Hourly frequency and mean duration of reflux episodes in the upright and supine period were also calculated in each patient. Symptoms (heartburn, acid regurgitation) were defined as being related to reflux if they started within five minutes of a fall in intraoesophageal $\mathrm{pH}$. The symptom index ${ }^{16}$ was calculated as the number of times the symptom occurred when the $\mathrm{pH}$ was below four, divided by the total number of times the symptom was reported; this quotient was then multiplied by 100 to give the percentage of symptoms associated with reflux. Intensity of symptoms was scored from mild $=1$ to severe $=3$ throughout the study; the duration of symptoms was evaluated at one minute intervals.

Comparisons between groups were performed with the Wilcoxon test for unpaired data and the Fisher test. Correlation were assessed with Spearman's rank correlation test.

\section{Results}

\section{GRADING OF PATIENTS}

Two groups of patients were identified by oesophageal manometry. Those with normal peristalsis (group I: $n=8$; mean age (SEM) 47 (3) years) were all women. Those with impaired peristalsis (group II: $n=8$; mean age (SEM) 48 (4) years; seven women, one man) had extremely low pressures in the oesophageal body and hypotonic lower oesophageal sphincter (Table I).

Endoscopy was normal in patients of group I, whereas all patients with impaired peristalsis had oesophagitis (grade 1, 2, or 3 in four, two, and two patients respectively). The severity of reflux symptoms were not statistically different between groups I and II (Table I), but heartburn, dysphagia, and regurgitation were more common in patients with impaired peristalsis and oesophagitis. 


\section{ACID PERFUSION TEST}

The intraoesophageal $\mathrm{pH}$ was not different in groups I and II during saline and acid perfusion. After perfusion, the acid clearance time (mean $($ SEM)) was significantly shorter $(\mathrm{p}<0.01)$ in patients with normal peristalsis (173 (28) seconds) than in those with impaired peristalsis (445 (48) seconds) (Fig 1). The number of swallows in the 10 minutes during and after acid infusion, calculated from the number of detectable oesophageal contractions in the most cranial recording channel, did not differ significantly in group I (20 (3); 20 (4)) or group II (18 (5); $18(6))$.

\section{OVERNIGHT PH MONITORING}

Overnight exposure of the oesophagus to acid, the number of reflux episodes longer than five minutes, and the duration of the longest period of reflux were significantly greater in patients with impaired peritalsis. The total number of reflux episodes, however, did not differ significantly between the groups (Table II). Frequency of reflux episodes did not differ between the groups in the upright and supine period, whereas the length of reflux was significantly longer in the supine period in patients with impaired peristalsis (Fig 2).

Figure 3 shows the relation between acid clearance time after acid perfusion and overnight exposure to acid. Acid clearance time was significantly correlated to acid exposure time (Spearman's $r=0.82 ; p<0.01$ ). Cut off values for acid clearance times of 290 seconds and for the percentage of time at $\mathrm{pH}<4$ of $11 \cdot 5 \%$ discriminated in all but one patient between groups $I$ and II.

\section{ACID SENSITIVITY}

Only three of 16 patients did not report a history of heartburn or acid regurgitation during the study period; all three had normal peristalsis and no oesophagitis. Two patients with impaired peristalsis and oesophagitis reported a history of symptoms but not during the study period. Heartburn or acid regurgitation were reported only when the oesophageal $\mathrm{pH}$ was less than four - that is, the symptom index was always $100 \%$ and was independent of endoscopic changes in the oesophageal mucosa and oesophageal function.

The nature, duration, and severity of symp-

TABLE I Clinical and investigational features of patients with progressive systemic sclerosis

\begin{tabular}{|c|c|c|c|}
\hline & $\begin{array}{l}\text { Group I }(n=8) \\
\text { Normal oesophageal } \\
\text { peristalsis }\end{array}$ & $\begin{array}{l}\text { Group II }(n=8) \\
\text { Impaired oesophageal } \\
\text { peristalsis }\end{array}$ & $p$ \\
\hline Dysphagia & 3 & 5 & NS \\
\hline Heartburn & 2 & 6 & NS \\
\hline Regurgitation & 1 & 4 & NS \\
\hline Nausea & 4 & 0 & NS \\
\hline Vomiting & 0 & 1 & NS \\
\hline \multicolumn{4}{|l|}{ Distal oesophageal contractions } \\
\hline Amplitude (mm Hg) & $77(8)$ & $3(2)$ & $<0.01$ \\
\hline $\begin{array}{l}\text { Duration (sec) } \\
\text { LOS pressure }(\mathrm{mm} H \mathrm{Hg})\end{array}$ & $4(0 \cdot 2)$ & $\star$ & \\
\hline \multicolumn{4}{|l|}{$L O S$ pressure $(\mathrm{mm} \mathrm{Hg})$} \\
\hline Slow pull through & $26(3)$ & $15(3)$ & $<0.05$ \\
\hline Rapid pull through & $36(2)$ & $20(3)$ & $<0.01$ \\
\hline
\end{tabular}

No of patients with symptoms are reported; pressures and durations are reported as mean (SEM); *not measurable as the shape of the pressure wave over the baseline was recognisable in only one patient; LOS=lower oesophageal sphincter.

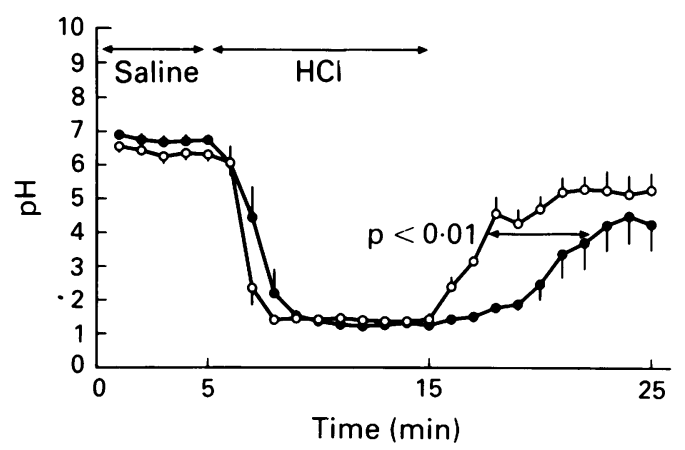

Figure 1: Intraoesophageal pH (mean (SEM)) in patients with progressive systemic sclerosis with normal peristalsis and no oesophagitis $(O)$ or with impaired peristalsis and oesophagitis (O) during infusion of saline, infusion of $\mathrm{HCl}$ $0 \cdot 1 \mathrm{~N}$ and after infusion. The difference in acid clearance time between the two groups was statistically significant.

toms did not differ between groups I and II during and after perfusion (Table III); the difference in the duration of symptoms, however, during the 10 minutes after perfusion almost reached conventional significance $(\mathrm{p}<0 \cdot 10)$. Symptoms during overnight $\mathrm{pH}$ monitoring lasted significantly longer $(p<0.05)$ in patients with impaired peristalsis and oesophagitis than in patients with normal peristalsis and no oesophagitis (Table III).

\section{Discussion}

These studies were performed in an unselected group of patients with PSS referred to our gastroenterology unit for the assessing of oesophageal involvement. Oesophagitis was seen only in the group with impaired peristalsis, confirming the close association between a change in oesophageal motility and injury of oesophageal mucosa. ${ }^{2}$

We measured the ability of the oesophagus to clear a given amount of acid; patients were allowed to swallow during and after acid perfusion. As expected, the acid clearance time was shorter in patients with normal peristalsis. Our experimental approach did not evaluate the effect of swallowing on the clearance of acid, but clearance was measured under conditions similar to those found during spontaneous reflux. Previous studies have recorded in normal volunteers ${ }^{17}$ and in patients with $\mathrm{PSS}^{7}$ the number of swallows at specific intervals that were needed to clear a given amount of acid placed in the oesophagus. We preferred to evaluate overall clearance of the oesophagus rather than the specific effects of swallowing because the amplitude of contractions after swallowing in patients with abnormal peristalsis is below the minimum effective strength for

TABLE II Twenty two hour intraoesophageal $p H$ in progressive systemic sclerosis

\begin{tabular}{lccc}
\hline & Group I & Group II & \\
\hline Percentage of time at $\mathrm{pH}<4$ & $5 \cdot 8(2 \cdot 3)$ & $30 \cdot 0(6.0)$ & $<0.01$ \\
Minutes at $\mathrm{pH}<4$ & $64(29)$ & $385(8)$ & $<0.01$ \\
No of reflux episodes & $21(4)$ & $36(6)$ & $\mathrm{NS}$ \\
No of episodes $>5$ minutes & $3(1)$ & $8(1)$ & $<0.01$ \\
Longest episode (min) & $40(24)$ & $118(16)$ & $<0.05$
\end{tabular}

Group I: patients with normal peristalsis + no oesophagitis; group II: patients with impaired peristalsis +oesophagitis; data are mean (SEM). 

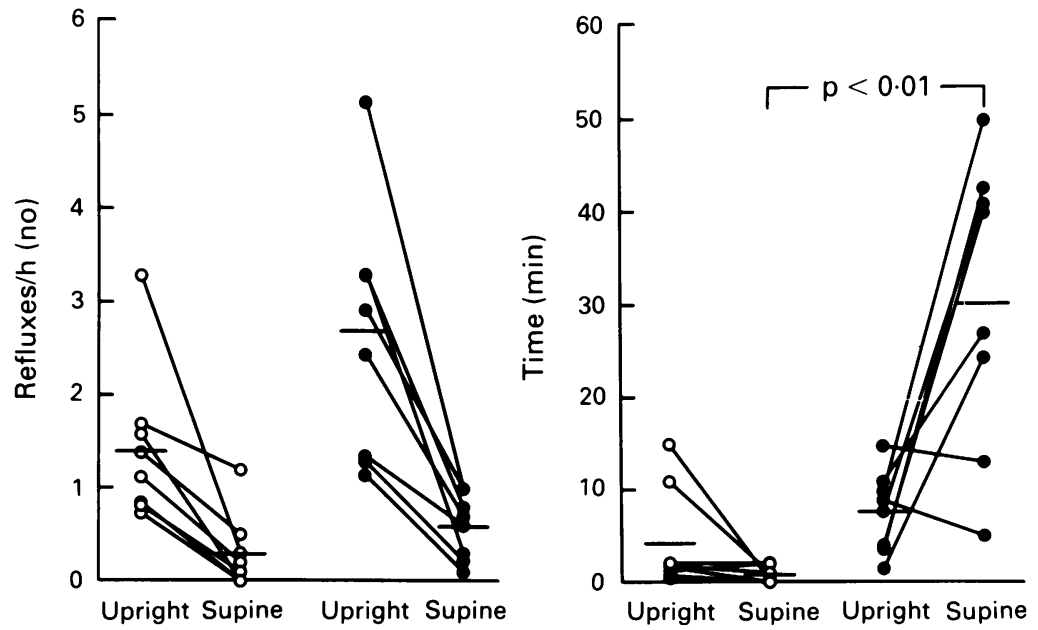

Figure 2: Frequency and duration of reflux episodes during overnight $p H$ monitoring in progressive systemic sclerosis patients with normal peristalsis and no oesophagitis $(O)$ or with impaired peristalsis and oesophagitis (O). Horizontal bars show the means.

clearance. ${ }^{18}$ In any event, the number of swallows did not differ in patients with or without peristalsis. Further studies will be needed to establish the relative roles of gravity, residual motility, and saliva as determinants of oesophageal clearance in PSS.

In our experimental conditions, acid clearance time discriminated between patients with normal and impaired peristalsis; moreover, acid clearance time was correlated with the overnight acid exposure time. These results are consistent with scintigraphic evidence of a delay in oesophageal transit of a radiolabelled liquid meal in PSS patients with impaired peristalsis ${ }^{3}$ and of fewer but more prolonged episodes of postprandial reflux when PSS patients were compared with those with normal peristalsis and equally severe oesophagitis. ${ }^{4}$ Thus, acid clearance times can be used to assess the impairment of the oesophageal motor function in PSS.

We also studied oesophageal sensitivity to acid during acid perfusion test and overnight $\mathrm{pH}$ monitoring. Our hypothesis was that the oesophageal sensitivity to acid would be reduced in PSS with a peristalsis on the basis of changed oesophageal sensory pathways. Patients were rather specific in their reporting of symptoms as they complained of heartburn or acid regurgitation only when the intraoesophageal $\mathrm{pH}$ was less than four. A substantial percentage of patients, greater than that previously reported in PSS, complained of heartburn or acid regurgitation during acid perfusion. Moreover, the nature, duration, and severity of symptoms during acid perfusion were similar in patients with or with-

Figure 3: Relation between percentage of time with an intraoesophageal $\mathrm{pH}<4$ during overnight $p H$ monitoring and acid clearance time in progressive systemic sclerosis. Patients with normal peristalsis and no oesophagitis (group I: O); those with impaired peristalsis and oesophagitis (group II: O). Line values discriminated all but one patient of group I from those of group II.
TABLE III Symptoms of heartburn and acid regurgitation in patients with progressive systemic sclerosis

\begin{tabular}{|c|c|c|c|}
\hline & Group I & Group II & \\
\hline \multicolumn{4}{|l|}{ During acid perfusion test } \\
\hline Patients with symptoms & $5 / 8$ & $5 / 8$ & NS \\
\hline Minutes before symptoms & $4(1 \cdot 4)$ & $3.6(1.4)$ & NS \\
\hline Minutes with symptoms & $4(1)$ & $4(1)$ & NS \\
\hline Severity of symptoms & $1 \cdot 4(0 \cdot 5)$ & $1 \cdot 0(0 \cdot 4)$ & NS \\
\hline \multicolumn{4}{|c|}{ During the 10 minutes after the acid perfusion test } \\
\hline Patients with symptoms & $5 / 8$ & $5 / 8$ & NS \\
\hline Minutes with symptoms & $1 \cdot 5(0.6)$ & $2 \cdot 5(1 \cdot 2)$ & $<0 \cdot 10$ \\
\hline Severity of symptoms & $0.6(2)$ & $0.6(2)$ & NS \\
\hline \multicolumn{4}{|l|}{ During 22 hour $\mathrm{pH}$ monitoring } \\
\hline Patients with symptoms & $2 / 8$ & $6 / 8$ & $\mathrm{~N}$ \\
\hline Minutes with symptoms & $3(2)$ & $50(23)$ & $<0.05$ \\
\hline Severity of symptoms & $0 \cdot 3(0 \cdot 2)$ & $1 \cdot 0(0 \cdot 3)$ & $<0 \cdot 10$ \\
\hline
\end{tabular}

Group I: patients with normal peristalsis + no oesophagitis; group II: patients with impaired peristalsis + oesophagitis; continuous measures are reported as mean $(\mathrm{SEM}) ; \mathrm{NS}=\mathrm{p}>0 \cdot 10$.

out impaired peristalsis. Thus, oesophageal sensitivity to acid was preserved in our patients.

Symptoms during overnight $\mathrm{pH}$ monitoring lasted longer in patients with prolonged oesophageal acid exposure. This is consistent with the positive correlation between the frequency and duration of gastro-oesophageal reflux symptoms and oesophageal acid exposure in dyspeptic patients with or without oesophagitis. ${ }^{19}$ Our PSS patients reported reflux symptoms, however, only during a small fraction of the acid exposure time, as previously seen in patients with chest pain ${ }^{20}$ or gastro-oesophageal reflux..$^{21}$ Symptoms of reflux therefore reflected poorly acid exposure times in patients with PSS as they do in patients with reflux and no connective tissue diseases. The discrepancy between the exposure time to acid and the extent of symptoms was striking in PSS, perhaps because of the prolonged oesophageal acid exposure in these patients, particularly during the supine period.

In summary, our studies of unselected patients with PSS show that impaired oesophageal peristalsis delays the clearance of acid and increases the exposure time to acid. Acid clearance time is a useful index of impaired oesophageal motor function. The assessment of the acid clearance time could be a useful alternative to the more time consuming and uncomfortable overnight evaluation of $\mathrm{pH}$. Despite the reliable reporting of symptoms and conserved sensitivity to acid, reflux symptoms reflected poorly the prolonged oesophageal acid exposure and do not seem to be a reliable guide to the severity of oesophageal involvement in PSS.

The authors thank Dr S F Phillips for helpful discussion of the manuscript and Ms Lois Law for preparing the manuscript.

1 Garrett JM, Winkelmann RK, Schlegel JF, Code CF Esophageal deterioration in scleroderma. Mayo Clin Proc 1971; 46: 92-6.

2 Zamost BJ, Hirschberg J, Ippoliti AF, Furst DE, Clements PJ, Weinstein WM. Esophagitis in scleroderma. Prevalence and risk factors. Gastroenterology 1987; 92: 421-8.

3 Klein HA, Wald A, Graham TO, Campbell WL, Steen VD. Comparative studies of esophageal function in systemic sclerosis. Gastroenterology 1992; 102: 1551-6.

4 Murphy JR, McNally P, Peller P, Shay SS. Prolonged clearance is the primary abnormal reflux parameter in patients with progressive systemic sclerosis and esophagitis. Dig Dis Sci 1992; 37: 833-41.

5 Maddern GJ, Horowitz M, Jamieson GG, Chatterton BE, Collins PJ, Roberts-Thomson P. Abnormalities of esophageal and gastric emptying in progressive systemic sclerosis. Gastroenterology $1984 ; 87: 922-6$.

6 Dodds WJ, Hogan WJ, Helm JF, Dent J. Pathogenesis of reflux esophagitis. Gastroenterology 1981; 81: 376-94.

7 Orringer MB, Dabich L, Zarafonetis CJD, Sloan H. Gastroesophageal reflux in esophageal scleroderma: diagnosis and implications. Ann Thorac Surg 1976; 22: 120-30. 
8 Gordon RM, Silverstein A. Neurologic manifestations in progressive systemic sclerosis. Arch Neurol 1970; 22: 12634.

9 Di Trapani G, Tulli A, La Cara A, Laurienzo P, Mazza S, David P. Peripheral neuropathy in course of progressive

Sonnex C, Paice E, White AG. Autonomic neuropathy in systemic sclerosis: a case report and evaluation of six systemic sclerosis: a case report and
patients. Ann Rheum Dis 1986; 45: 957-60

11 Klimiuk PS, Taylor L, Baker RD, Jayson MIV. Autonomic neuropathy in systemic sclerosis. Ann Rheum Dis 1988; 47: 542-5.

12 Berth-Jones J, Coates PAA, Graham-Brown RAC, Burns DA. Neurological complications of systemic sclerosis - a report of three cases and review of the literature. Clin Exp Dermatol $1990 ; 15: 91-4$

13 Cohen S, Fisher R, Lipshutz W, Turner R, Myers A, Schumacher $\mathbf{R}$. The pathogenesis of esophageal dysfunction in scleroderma and Raynaud's disease. F Clin Invest 1972; 51: 2663-8.

14 Masi AT. Preliminary criteria for the classification of systemic sclerosis (scleroderma). Arthritis Rheum 1980; 23: 581-9.

15 Richter JE, Wu WC, Johns DN, Blackwell JN, Nelson JL,
Castell JA, et al. Esophageal manometry in 95 healthy adult volunteers. Variability of pressures with age and frequency of 'abnormal' contractions. Dig Dis Sci 1987; 32: 583-92.

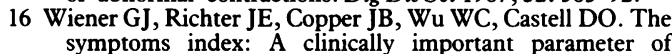
ambulatory 24-hour esophageal pH monitoring. $\mathrm{Am} \mathcal{F}$ Gastroenterol 1988; 83: 358-61.

17 Helm JF, Dodds WJ, Riedel DR, Teeter BC, Hogan WJ, Arndorfer RC. Determinants of esophageal acid clearance in normal subjects. Gastroenterology 1983; 85: 607-12.

18 Kahrilas PJ, Dodds WJ, Hogan WJ. Effect of peristaltic dysfunction on esophageal volume clearance. Gastrodysfunction on esophageal
enterology $1988 ; 94: 73-80$.

19 Joelsson B, Johnsson F. Heartburn - the acid test. Gut 1989; 30: $1523-5$

20 Breumelhof $\mathrm{R}$, Nadorp JHSM, Akkermans LMA, Smout AJPM. Analysis of 24-hour esophageal pressure and $\mathrm{pH}$ data in unselected patients with noncardiac chest pain. Gastroenterology 1990; 99: 1257-64.

21 Baldi F, Ferrarini F, Longanesi A, Ragazzini M, Barbara L. Acid gastroesophageal reflux and symptom occurrence. Analysis of some factors influencing their association. Dig Dis Sci 1989; 34: 1890-3. 